\title{
Chronic complicated osteomyelitis of the appendicular skeleton: diagnosis with technetium-99m labelled monoclonal antigranulocyte antibody-immunoscintigraphy
}

\author{
Achim Kaim ${ }^{1,3}$, Thomas Maurer ${ }^{2}$, Peter Ochsner², Gernot Jundt, Eberhard Kirsch ${ }^{3}$, Jan Mueller-Brand ${ }^{1}$ \\ 1 Institute of Nuclear Medicine, University Hospital Basel, Switzerland \\ 2 Department of Orthopedic and Trauma Surgery, Kantonsspital Liestal, Switzerland \\ ${ }^{3}$ Institute of Diagnostic Radiology, University Hospital Basel, Switzerland \\ ${ }^{4}$ Institute of Pathology, University Hospital Basel, Switzerland \\ Received 6 January and in revised form 5 April 1997
}

\begin{abstract}
Chronic post-traumatic osteomyelitis (OM) represents a particular challenge for nuclear medicine and radiology since clinical and biochemical parameters are frequently unreliable. The aim of this study was to investigate the value of combined bone scan (BS) and immunoscintigraphy (IS) with technetium-99m labelled monoclonal antigranulocyte antibody (MAB) in patients with suspected chronic OM of the appendicular skeleton. Twenty-four patients (17 females and 7 males) with suspected chronic post-traumatic OM were evaluated with three-phase BS/ $99 \mathrm{~m}$ Tc-MAB-IS. The final diagnosis was established by means of bone culture and histology in 19 cases and clinical follow-up in five cases. The studies were reviewed by two independent and experienced observers; the interobserver agreement was calculated by kappa statistics. The sensitivity, specificity and accuracy of BS alone were $92 \%, 18 \%$ and $58 \%$, respectively. Combined BS/ $99 \mathrm{mTc}-\mathrm{MAB}-\mathrm{IS}$ had a sensitivity, specificity and accuracy of $84 \%, 72 \%$ and $79 \%$, respectively. Of 24 studies, 11 were true-positive, two false-negative, eight true-negative and three false-positive. Two patients presented with unexpected ectopic haematopoietic bone marrow in the appendicular skeleton that caused falsepositive results. A high degree of interobserver agreement was found $(\kappa=0.85)$. It is concluded that combined $\mathrm{BS} / 99 \mathrm{~m} \mathrm{Tc}-\mathrm{MAB}-\mathrm{IS}$ represents a very sensitive and reproducible method with an acceptable specificity for the investigation of chronic OM. Problems may occur in the differentiation of low-grade OM from aseptic inflammation. Another problem is ectopic marrow that may occur in the appendicular skeleton due to a chronic inflammatory stimulus. A former intramedullary intervention in the femur with displacement of haematopoietic marrow may also lead to an ectopic location.
\end{abstract}

Correspondence to: A. Kaim, Institute of Nuclear Medicine, University Hospital Basel, Petersgraben 4, CH-4032 Basel, Switzerland
Eur J Nucl Med (1997) 24:732-738

\section{Introduction}

Chronic post-traumatic and postoperative osteomyelitis represents a disabling disease that requires a detailed investigation for assessment of active infection and local extent in order to decide upon the appropriate therapeutic management. Beside radiological methods [X-ray plain film, computed tomography (CT), magnetic resonance imaging (MRI)], nuclear medicine offers a wide variety of radiopharmaceuticals for the imaging of inflammation or infection, e.g. gallium-67 citrate $[1,2]$, technetium-99m labelled nanocolloids [3], $99 \mathrm{mTc}-1 \mathrm{a}-$ belled human immunoglobulins [4-6], indium-111 oxine and $99 \mathrm{~m}$ Tc-hexamethylpropylene amine oxime (HMPAO)-labelled autologous leucocytes [7-11]. ${ }^{99 \mathrm{~m} T \mathrm{~T}-}$ labelled monoclonal antigranulocyte antibody (MAB) immunoscintigraphy (IS) represents a simple, practicable and specific method that has been extensively studied for the detection of inflammatory and septic processes. Extra-osseous infection [12, 13], acute and subacute osteomyelitis [14-16], spondylodiscitis [6, 14] and infection of hip arthoplasties $[12,13,16,17]$ have been the main subjects of these publications. Until now no study has specifically considered the value of IS in chronic recurrent or low-grade osteomyelitis of the appendicular skeleton. Therefore, the accuracy of IS with MAB as an adjunct to bone scan in the evaluation of this rare but important patient group was assessed.

\section{Materials and methods}

Patients Twenty-four patients (17 males and 7 females; mean age 47 years, range $21-83$ years) referred to the Nuclear Medicine de- 
partment with suspected bone infection were examined with bone scan and IS. Only patients with suspected chronic osteomyelitis of the appendicular skeleton were included. Arthroplasties of the hip were not considered. One or more of the following criteria was present: clinical signs (swelling, heat, redness, fistula), pain, fever, elevated laboratory parameters (ESR, CRP, WBC count) and suspected focal infection on radiology (X-ray plain film, CT, MRI). All patients suffered from longstanding, post-traumatic chronic osteomyelitis; the mean interval from first surgery to the present study was 8.6 years (range 2 months to 42 years). The average number of operations due to recurrent osteomyelitis was four (range 1-15), and the interval from last surgery to the present averaged 3.5 years ( 2 months to 42 years). Six patients had orthopaedic devices (metal plate, intramedullary nail, external fixator). The suspected areas were investigated by X-ray plain films in all patients, by CT in 11 patients and by MRI in nine patients. Final diagnosis was established by surgery with microbiological and/or histological analysis in 19 patients and by clinical course over a period of at least 6 months in five patients. Radiological results were considered in defining the final diagnosis.

Radiopharmaceuticals The antigranulocyte antibody used for IS is a murine monoclonal antibody (BW250/183), supplied by Behringwerke $A G$, Switzerland. The antibody is an immunoglobulin of the IgG isotype and binds to the epitope NCA 95 on the surface of human granulocytes. The antibody is reduced with stannous IIchloride solution and labelled with ${ }^{99 \mathrm{mT}} \mathrm{c}$. After an incubation period of $10 \mathrm{~min}, 555 \mathrm{MBq}$ of the antibody solution (equivalent to
$0.5 \mathrm{mg}$ of the antibody) was intravenously injected into the patient. Serum was obtained before and 3 weeks after injection to determine the titre of human antimouse antibodies (HAMA). None of the patients developed HAMA, and no side-effects or adverse reactions were observed.

For ${ }^{99} \mathrm{mTc}$-diphosphonate bone scan, 3,3-diphosphono-1,2-propanedicarboxyl acid tetrasodium salt (DPD) (Hoechst, Switzerland) was used. It was labelled with an average of $740 \mathrm{MBq}$ of $99 \mathrm{mTc}$.

Imaging modalities All patients first underwent quantitative threephase bone scan followed by IS at an interval of 2 days to 1 week. After injection of $99 \mathrm{~m}$ Tc-DPD 205 -s images were immediately registered and another 60 -s image was taken at $5 \mathrm{~min}$. Five hundred-kcount planar spot images were obtained $3 \mathrm{~h}$ after injection and stored on computer using a $256 \times 256$ matrix.

IS was performed $17 \mathrm{~h}$ p.i. Planar spot images of the suspected area in at least two projections were obtained. Five hundredkcount images with a $256 \times 256$ matrix were recorded. Additionally whole-body images were obtained. Imaging was performed with a Siemens DIACAM gamma camera equipped with a low-energy high-resolution (LEHR) collimator connected to a dedicated computer system (Icon, Siemens).

Data interpretation Bone scans and IS were reviewed by two experienced observers (A, B) unaware of the definitive diagnosis. The readers were independent of each other and interpreted the studies separately. X-ray plain films were used to identify the
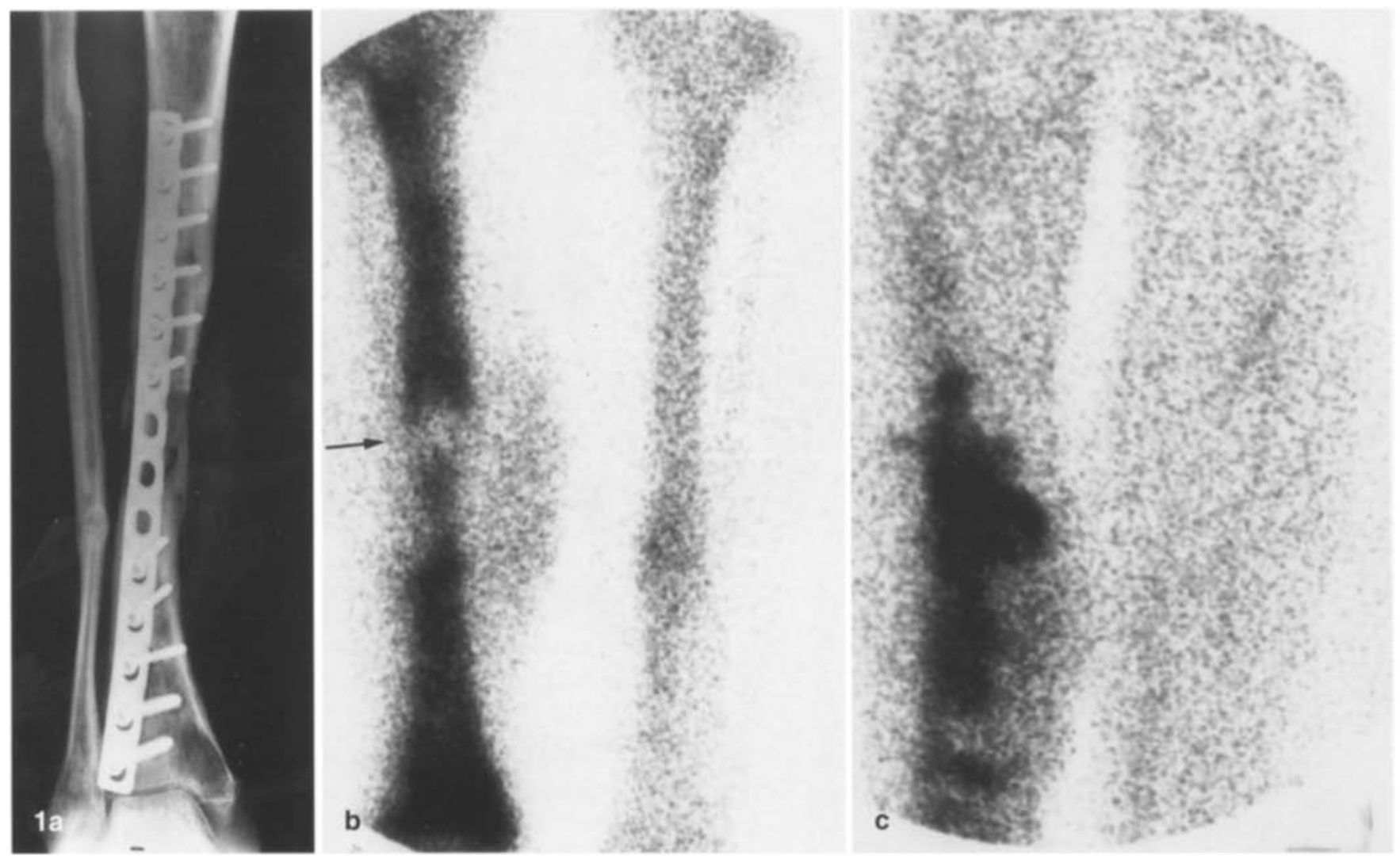

Fig. 1a-c. True-positive result in a patient with infected pseudarthrosis and bone necrosis 3 years after trauma. Plate osteosynthesis had been performed 1 year prior to the current investigation. a Radiograph (anteroposterior) shows pseudarthrosis of the tibial diaphysis. b Late bone scan (anterior) shows hyperactivity of the complete tibia with a photopenic zone at the site of pseudarthrosis suggesting bone necrosis (black arrow). $\mathbf{c}$ Intense accumulation of $99 \mathrm{mTc}-\mathrm{MAB}$ on IS (anterior) demonstrating the extent of the infection 
anatomy. The degree of agreement between the two observers was measured by kappa statistics [18] calculating the index $\kappa$ ( $\kappa$ is 0.0 when there is just a chance agreement and 1.0 when there is perfect agreement). Two of 26 studies were interpreted differently and had to be discussed with a third observer (C). After analysing these cases, agreement was achieved among all three observers and the final scintigraphic diagnosis was established. These data were the basis for calculating sensitivity, specificity and accuracy.

IS was considered positive when accumulation was observed in comparison to background and the contralateral side. Uptake was graded as absent, mild or strong. Normal late bone scan and positive IS were interpreted as indicating soft tissue infection. Osteomyelitis was diagnosed if bone scan and IS were positive at the same anatomical location. Infected necrotic bone was considered in zones of decreased bone metabolism with positive IS.

\section{Results}

Twenty-four patients with suspected osteomyelitis were examined. Thirteen patients presented with active osteomyelitis, four had soft tissue infections and in the re- maining seven surgery, histology, cultures radiology and/or clinical course failed to reveal evidence of infection. Of the 24 suspected lesions, 15 were located in the tibia, eight in the distal femur and one in the diaphysis of the humerus. Laboratory results (ESR, CRP, WBC count) showed elevated levels in six patients with active osteomyelitis, in four patients with soft tissue infection and in one patient without infection. The remaining patients (seven with osteomyelitis, six without osteomyelitis) had normal laboratory results. In $9 / 13$ patients the causative micro-organism was Staphylococcus aureus, in 3/13 patients coagulase-negative Staphylococcus and in $1 / 13$ patients diagnosis was confirmed by histology without microbiology. Delayed bone scan $(3 \mathrm{~h})$ revealed increase bone metabolism in 21/24 cases. In 3/21 cases areas of photopenia occurred beside zones of increased tracer activity corresponding to necrotic bone. Considering the bone scan alone, sensitivity, specificity and accuracy were calculated to be $92 \%, 18 \%$ and $58 \%$, respectively. Combined bone scan and IS had a sensitivity,
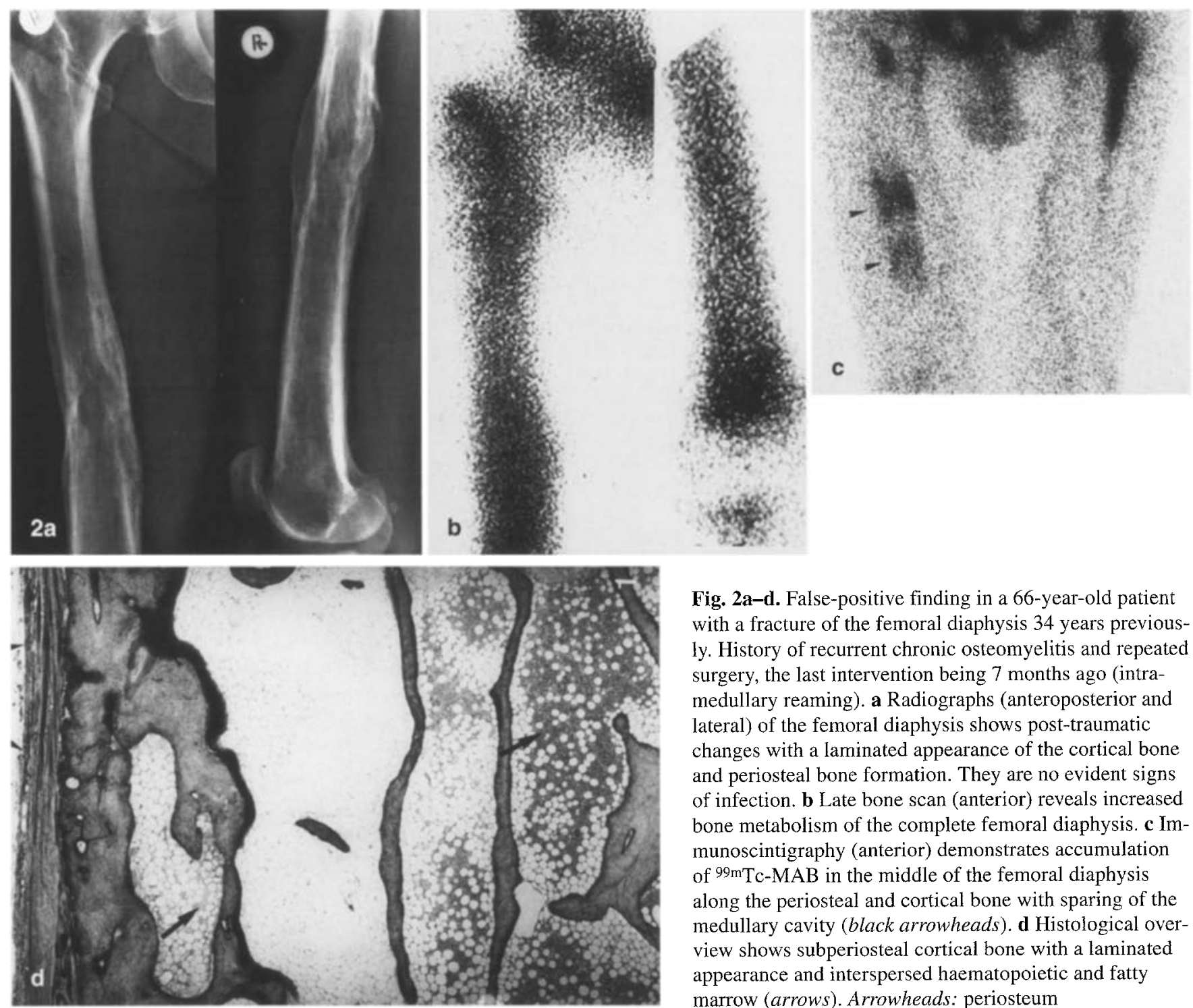

Fig. 2a-d. False-positive finding in a 66-year-old patient with a fracture of the femoral diaphysis 34 years previously. History of recurrent chronic osteomyelitis and repeated surgery, the last intervention being 7 months ago (intramedullary reaming). a Radiographs (anteroposterior and lateral) of the femoral diaphysis shows post-traumatic changes with a laminated appearance of the cortical bone and periosteal bone formation. They are no evident signs of infection. b Late bone scan (anterior) reveals increased bone metabolism of the complete femoral diaphysis. $\mathbf{c ~ I m - ~}$ munoscintigraphy (anterior) demonstrates accumulation of $99 \mathrm{mTc}-\mathrm{MAB}$ in the middle of the femoral diaphysis along the periosteal and cortical bone with sparing of the medullary cavity (black arrowheads). d Histological overview shows subperiosteal cortical bone with a laminated appearance and interspersed haematopoietic and fatty marrow (arrows). Arrowheads: periosteum 
Table 1. Individual patient data

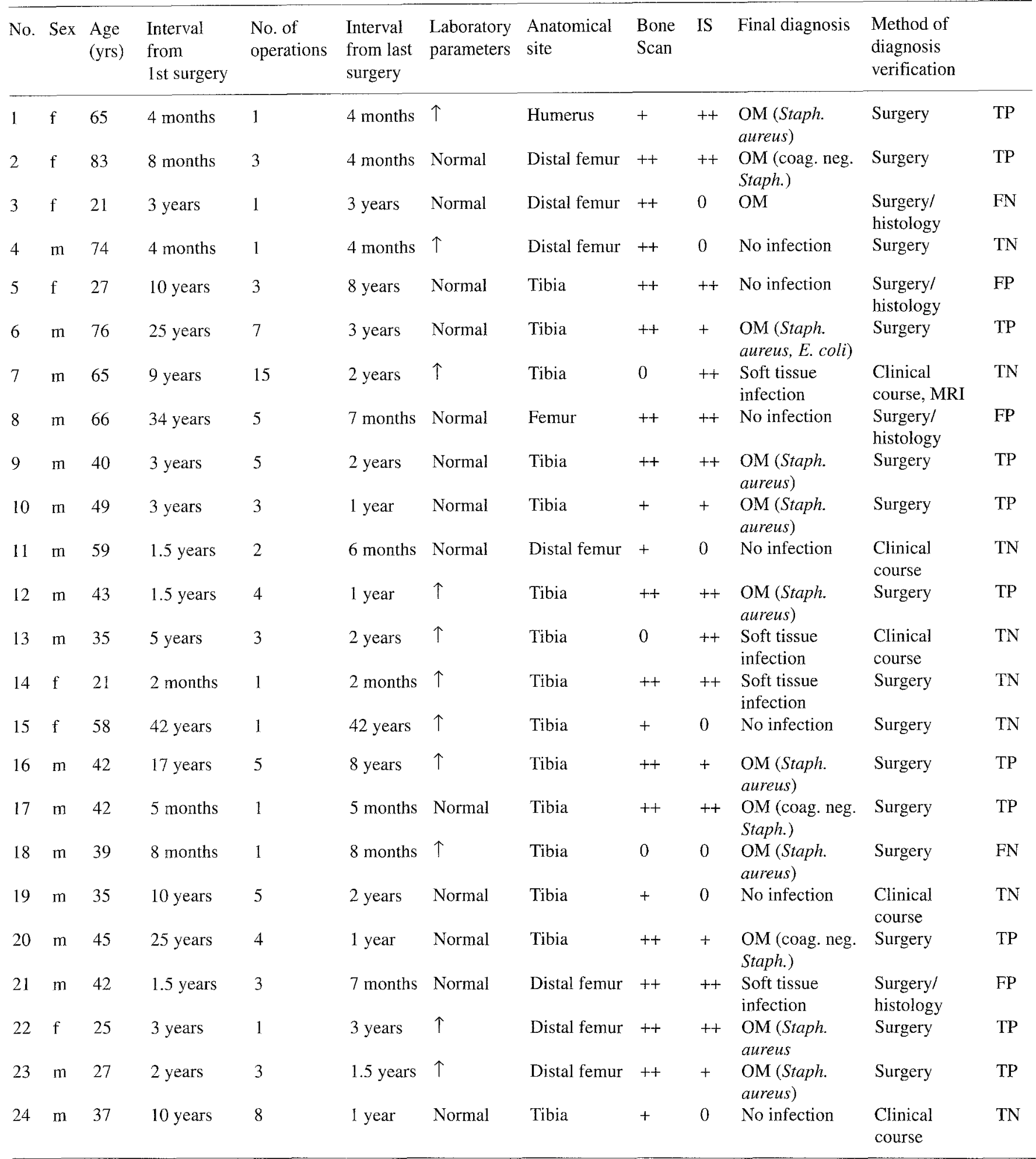

0, No uptake; +, mild uptake; ++, strong uptake; TP, true-positive; FN, false-negtive; TN, true-negative; FP, false-positive

specificity and accuracy of $84 \%, 72 \%$ and $79 \%$, respectively. Of the 24 studies, 11 were true-positive (TP) and eight, true-negative (TN). The scintigraphic pattern that occurred in active osteomyelitis with infected pseudarthrosis and necrotic bone is demonstrated in Fig. 1. Two studies were false-negative (FN) and three, false-positive
(FP). Figure 2 illustrates an FP finding that is discussed later.

There was a strong agreement between the two observers $\mathrm{A}$ and $\mathrm{B}$. The $\mathrm{k}$-value for interobserver agreement was 0.85 (95\% confidence interval 0.74-0.99).

The individual patient data are summarized in Table 1. 


\section{Discussion}

The diagnosis of chronic complicated osteomyelitis represents a particular challenge for radiology and nuclear medicine. Low-grade and recurrent longstanding infection may cause chronic pain, fistulae and bone necrosis with pathological fracture. The disease may lead to disability and impair quality of life in a young patient population. Frequently clinical and biochemical parameters are inadequate for making the diagnosis. Our series confirms that laboratory parameters are inconclusive in the evaluation of chronic osteomyelitis. The assessment of disease activity, disease extension and the separation of bony from soft tissue infection influence the therapeutic management by the referring surgeon. Nuclear medicine offers very useful information in the investigation of bone infection. ${ }^{99 m} \mathrm{mc}$-diphosphonate bone scan represents the basic study and is very sensitive in detecting bone alterations. However, it is unable to distinguish between infection and aseptic post-traumatic changes; thus, we calculated a specificity of $18 \%$. Diphosphonate scan has to be followed by inflammatory scintigraphy, and radiolabelled autologous leucocyte scintigraphy and ${ }^{99 m}$ Tc-labelled MAB-IS are specific for the detection of infected foci. The clinical use of these methods is predominantly determined by their potential availability and their practicability in routine clinical practice. Radiolabelled autologous white cells are still considered the gold standard in the nuclear imaging of infected sites. However, the examination requires time-consuming ex vivo blood handling techniques and special training, and entails risk of infection for both laboratory personal and patients.

99m Tc-MAB binding granulocytes show a quite similar in vivo distribution to that of radiolabelled granulocytes and allow the detection of granulocyte accumulation in the appendicular skeleton $[19,20]$. The advantage of $99 \mathrm{mTc}-\mathrm{MAB}$ is the simplicity of the labelling process of Tc with the antibodies and the in vivo labelling of granulocytes. The antibodies are directed against the myeloidspecific surface antigen NCA-95 expressed by granulocytes. A disadvantage of MAB is the induction of HAMA in $3 \%$, which can lead to altered biodistribution $[21,22]$. Allergic reactions or serious adverse reactions have not been reported so far. Problems in the detection of infection appear in areas of active bone marrow such as the spine, the pelvis and the proximal femora because precursor cells of the granulopoietic system such as promyelocytes are also labelled. A physiological uptake in the bone marrow occurs and infection causes non-specific photopenic zones due to bone marrow destruction. All patients included in our study had suspected infections in the appendicular skeleton. Chronic infection of the pelvis and spine were excluded, as was hip prosthesis infection. Sensitivity, specificity and accuracy for proven infection were calculated to be $84 \%, 72 \%$ and $79 \%$ respectively.

Currently there are four published series with small numbers of patients reporting the use of ${ }^{99 \mathrm{~m}} \mathrm{Tc}-\mathrm{MAB}$ in chronic osteomyelitis [6, 14-16]. The reported sensitivity $(79 \%-100 \%)$ and specificity $(64 \%-100 \%)$ show a wide range due to the different inclusion criteria for chronic osteomyelitis. A major part of the reported data has been based on patient groups with acute and subacute infections $[6,14,15]$. Furthermore, patients with septic hip arthroplasties [15] and even chronic infection of the axial skeleton $[6,14]$ have been included. We considered only patients with suspicion of infection of the tubular bones at least 6 weeks after surgery or trauma, with the majority of patients having suffered from longstanding recurrent osteomyelitis. We therefore believe that the results of our study accurately reflect the ability to image infection with $\mathrm{MAB}$ in chronic post-traumatic osteomyelitis. The published studies reporting the use of 99mTc-HMPAO-labelled leucocytes for the detection of chronic osteomyelitis calculated values for sensitivity $(83 \%-93 \%)$ and specificitiy $(78 \%-100 \%)$ that suggest superiority over IS $[7,10,23,24]$. Until now, however, no comparative study that proves this hypothesis has been performed in this field of application.

There are several possible reasons for false-negative studies. One is technical problems such as incomplete labelling of the MAB. In follow-up studies there may be induction of HAMA that have the potential to intercept the antibody and therefore hinder specific accumulation at infection sites [22]. In our study no HAMA response was observed and the laboratory titre controls were normal. Another potential explanation is the inaccessibility of the infection site due to encapsulation. In osteomyelitis after intramedullary nailing an avascular zone in the central part of the diaphysis occurs due to hyperthermic injury and may be infected [25]. This infection between the nail and cortical bone may be encapsulated and thereby prevent the migration of the radiolabelled MABbinding granulocytes. One of our two false-negative studies is explained by this phenomenon. A further possible reason for equivocal findings is a small number of granulocytes at the infected site in chronic low-grade osteomyelitis. The histology of one patient with positive microbiology for infection revealed a chronic inflammation with predominantly lymphocytes and plasma cells and only some scattered granulocytes. IS did not show significant tracer accumulation and the study was finally considered to be false-negative.

On the other hand, non-purulent inflammation may sometimes include some polymorphonuclear cells which lead to a mild uptake on IS and cannot be distinguished from low-grade osteomyelitis. False-positive scans have been described in fractures, osteosarcoma, eosinophilic granuloma, Paget's disease and granulation tissue of all kinds $[14,26]$. Sometimes it is very difficult to determine whether infection is in bone or soft tissue. In one false-positive case, planar scintigraphy performed in two projections had been positive in bone scan and IS. Exact anatomical localization was impossible and the examinations were interpreted as positive for osteomyelitis. Surgery revealed a soft tissue infection without osseous in- 
volvement. Increased bone metabolism may be explained by the postoperative state and sometimes an overlying positive IS is of uncertain significance. This may lead to misinterpretation, and careful analysis of scintiscans and X-ray plain films for anatomical landmarks is necessary. In doubtful cases, single-photon emission tomography may be indicated.

Two patients presented with unexpected ectopic active bone marrow in the appendicular skeleton that gave rise to false-positive studies. In both cases histology revealed haematopoietically active bone marrow at the suspected locations. No histological signs of infection were found and microbiology was negative. The strongly positive IS was explained by the labelling of the promyelocytes with the ${ }^{99 \mathrm{~m} T c-M A B}$. One of the two patients had a 10-year history of recurrent post-traumatic osteomyelitis of the tibial diaphysis with repeated surgical procedures. The reason for this unexpected finding remains unclear. It is possible that various mediators such as cytokines, macrophage colony stimulating factor, granulocyte colony stimulating factor or bone morphogenetic protein [27-29] are released by stromal elements due to a chronic inflammatory stimulus and induce neogenesis of active bone marrow in the peripheral skeleton. Until now, however, there have been no reports of such a phenomenon, and further investigations are required. The other patient had a 34-year history of chronic osteomyelitis at the femoral middiaphysis that had been treated several times by medullary nailing or intramedullary reaming. Histology revealed islands of haematopoietically active bone marrow arranged in layers between the architecture of the remodelled cortical bone. One possible explanation for this case is that active bone marrow had been displaced from the proximal femur to the diaphyseal location during the intramedullary surgical procedures and integrated into cortical bone in the course of endosteal remodelling.

Two studies were differently interpreted by the two observers (A, B) and definitive diagnosis was established after discussion with a third observer. Both cases had been interpreted as negative by one observer due to mild radionuclide accumulation on IS which was thought to demonstrate a non-purulent inflammation. Considering the results of all examinations, however, a high degree of agreement was found between observer $A$ and $B(\kappa=0.85)$, proving IS with MAB to be a reproducible method for the recognition of chronic osteomyelitis.

Two recently published reports have presented encouraging results using a $99 \mathrm{~m} \mathrm{Tc}$-antigranulocyte monoclonal antibody fragment $[10,24]$. The advantages over $\mathrm{MAB}$ are the shortened imaging time and a negligible HAMA response rate. The sensitivity, specificity and accuracy were comparable or even superior to those ob-

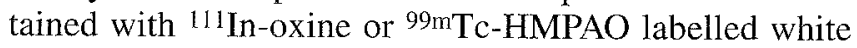
blood cells. Further studies to assess the clinical value of this imaging method in chronic osteomyelitis are being planned.
In conclusion, this study confirms that IS is a very sensitive, accurate and reproducible method for evaluating chronic complicated osteomyelitis. We found the specificity to be only $18 \%$ with bone scan alone whereas combined bone scan and IS yielded a specificity of $72 \%$. One major problem is the differentiation between lowgrade infection and aseptic inflammation; a further problem derives from the ectopic active bone marrow that may occur in the appendicular skeleton due to chronic inflammation or former surgical procedures, resulting in false-positive studies. Image interpretation should be performed with X-ray plain film since disturbed postoperative anatomy may exist, and one should be aware of the patient's history and former surgical procedures. The majority of patients represent complicated cases with limitations on activities of daily life. Therefore preoperative assessment utilizing the modern imaging modalities of nuclear medicine (bone scan, IS) and radiology (CT, $\mathrm{MRI}$ ) is required, with the modalities being used in a complementary fashion.

\section{References}

1. Sorsdahl OA, Goodhart GL, Williams HT, Hanná LJ, Rodriquez J. Quantitative bone gallium scintigraphy in osteomyelitis. Skeletal Radiol 1993; 22: 239-242.

2. Al-sheikh W, Sfakianokis GN, Mnaymnehz W, et al. Subacute and chronic bone infection: diagnosis using ${ }^{111} \mathrm{In},{ }^{67} \mathrm{Ga}$ and 99mTc-MDP bone scintigraphy and radiography. Radiology 1985; 155: 501-506.

3. Flivik G, Sloth M, Rydholm U, Herrlin K, Lidgren L. ${ }^{99 m T c-}$ nanocolloid scintigraphy in orthopedic infections: a comparison with ${ }^{111}$ In-labeled leukocytes. J Nucl Med 1993; 34: 1646-1650.

4. Datz FL, Anderson CE, Ahluwalia R, et al. The efficiency of $11 \mathrm{In}$-polyclonal $\mathrm{IgG}$ for the detection of infection and inflammation. J Nucl Med 1994; 35: 74-83.

5. Ang ES, Sundram FX, Goh ASW. $99 \mathrm{mTc}$-polyclonal IgG and $99 \mathrm{mTc}$-nanocolloid scans in orthopedics: a comparison with conventional bone scan. Nucl Med Commun 1993; 14: 419-432.

6. Sciuk J, Brandau W, Vollet B, Stiicker R, Erlemann R, Bartenstein P, Peters PE, Schober O. Comparison of $99 \mathrm{~m}$ Tc polyclonal human immunoglobin and ${ }^{99 \mathrm{~m}} \mathrm{Te}$ monoclonal antibodies for imaging chronic osteomyelitis. First clinical results. Eur $J$ Nucl Med 1991; 18: 401-407.

7. Esper EL, Dacquet V, Paillard J, Bascoulergue G, Talion MM, Fonroget J. ${ }^{99 m} \mathrm{Tc}-\mathrm{HMPAO}$-labeled leukocyte scintigraphy in suspected chronic osteomyelitis related to an orthopedic device: clinical usefulness. Nucl Med Commun 1992; 13: 799-805.

8. Roddie ME, Peters AM, Osman S, Danpure HJ, Lavender JP, Neirinckx RD. Osteomyelitis. Nucl Med Commun 1988; 9: 713-717.

9. Schauwecker DS. Osteomyelitis: diagnosis with ${ }^{11}$ In-labeled leucocytes. Radiology 1989; 171: 141-146.

10. Becker W, Bair J, Behr T, Repp R, Streckenbach H, Beck H, Gramatzki M, Winship MJ, Goldenberg DM, Wolf F. Detection of soft tissue infection and osteomyelitis using a ${ }^{99 \mathrm{~m} T c-1 a-}$ beled antigranulocyte monoclonal antibody fragment. $J \mathrm{NuCl}$ Med 1994; 35: 1436-1443. 
11. Seabold JE, Ferlic RJ, Mash JL, Nepola JV. Periarticular bone sites associated with traumatic injury: false positive findings with ${ }^{111}$ In-labeled white blood cell and $99 \mathrm{~m}$ Tc-MDP scintigraphy. Radiology 1993;186: 845-849.

12. Seybold K, Frey LD, Locher J. Immunoscintigraphy of infections using ${ }^{123 I}$ and ${ }^{99 m} \mathrm{mc}-$ labeled monoclonal antibodies. Advanced experiences in 230 patients. Angiology 1992; 43 : 85-90.

13. Seybold K, Locher J. Infektdiagnostik mit monoklonalen Antigranulozyten-Antikörpern. Therapiewoche Schweiz 1994; 10:275-283.

14. Hotze AL, Briele B, Overbeck B, Kropp J, Grünwald F, Mekkawy MA, von Smekal A, Möller F, Biersack HJ. ${ }^{99 \mathrm{~m}} \mathrm{Tc}-\mathrm{la}-$ beled antigranulocyte antibodies in suspected bone infections. I Nucl Med 1992; 33: 526-531.

15. Reuland P, Winkler KH, Heuchert T, Ruck R, MüllerSchauenburg W, Weller S, Feine U. Detection of infection in postoperative patients with ${ }^{99 \mathrm{~m} T \mathrm{c}-l a b e l e d}$ monoclonal antibodies against granulocytes. J Nucl Med 1991; 32: 2209-2214.

16. Scheidler J, Leinsinger G, Pfahler M, Kirsch CM. Diagnosis of osteomyelitis. Accuracy and limitations of antigranulocyte antibody imaging compared to three-phase bone scan. Clin Nucl Med 1994; 19: 731-737.

17. Boubaker A, Bischof Delaloye A, Blanc CH, Dutoit M, Leyvraz PF, Delaloye B. Immunoscintigraphy with antigranulocyte monoclonal antibodies for the diagnosis of septic loosening of hip prostheses. Eur J Nucl Med 1995; 22: 139-147.

18. Sachs L. Angewandte Statistik, 7 th edn. Berlin Heidelberg New York, Springer; 1991: 472-473.

19. Andres RY, Schubiger PA, Tiefenauer L, Seybold K, Locher JT, Mach JP, Buchegger F. Immunoscintigraphic localization of inflammatory lesions: concept, radiolablling and in-vitro testing of a granulocyte specific antibody. Eur $J \mathrm{Nucl} \mathrm{Med}$ 1988; 13: 582-586.
20. Becker W, Borst U, Fischbach W, Pasurka B, Schäfer R, Börner W. Kinetic data of in-vivo labeled granulocytes in humans with a murine ${ }^{99 m}$ Tc-labeled monoclonal antibody. Eur $J$ Nucl Med 1989; 15: 361-366.

21. Steinstraesser A, Oberhausen E. Granulocyte labelling kit BW 250/183. Result of the European multicenter trial. Nucl-Med 1996; 35: 1-11.

22. Hyams D, Reynolds JC, Carrasquillo JA, et al. The effect of circulating antimurine antibody on the pharmacokinetics and biodistribution of injected radiolabeled monoclonal antibody [abstract]. J Nucl Med 1986; 27: 922.

23. Ivancevic V, Dodig D, Livakovic M, Hancevic J, Ivancevic D. Comparison of three-phase bone scan, ${ }^{99 \mathrm{~m} T c-H M P A O ~ l e u k o-}$ cyte scan and ${ }^{67}$ gallium scan in chronic bone infection. Prog Clin Biol Res 1990; 355: 189-198.

24. Becker W, Palestro CJ, Winship J, Feld T, Pinsky CM, Wolf F, Goldenberg DM. Rapid imaging of infections with a monoclonal antibody fragment (LeukoScan). Clin Orthop 1996; 329: 263-272.

25. Ochsner PE, Brunazzi MG. Intramedullary reaming and soft tissue procedures in treatment of long bones. Orthopedics 1994; 17: 433-440.

26. Oyen WJG, van Horn JR, Claessens AMJ, Sloof TJJH, van der Meer JWM, Corstens FHM. Diagnosis of bone, joint and joint prosthesis infection with ${ }^{11}{ }^{1}$ n-labeled nonspecific human immunoglobulin. Radiology 1992; 182: 195-199.

27. Groopman JE, Molina JM, Scadden DT. Hematogenic growth factors: biology and clinical applications. N Engl J Med 1989; 321: 1449-1459.

28. Moore MAS. Clinical implications of positive and negative hematopoietic stem regulators. Blood 1991; 78: 1-19.

29. Kawai M, Hattori H, Yasue K, Mizutani H, Ueda M, Kaneda $\mathrm{T}$, Hoshino $\mathrm{T}$. Development of hemopoietic bone marrow within the ectopic bone induced by bone morphogenetic protein. Blood Cells 1994; 20: 191-191. 